\title{
Is There Any Correlation between Insulin Resistance and Nitrate Plasma Concentration in White Coat Hypertensive Patients?
}

\author{
Leila Maria Marchi-Alves and Evelin Capellari Carnio \\ Departamento de Enfermagem Geral e Especializada, Escola de Enfermagem de Ribeirão Preto/Universidade de São Paulo, \\ Avenida Bandeirantes, 3900, 14040-902-Ribeirão Preto, SP, Brazil \\ Correspondence should be addressed to Leila Maria Marchi-Alves, lmarchi@eerp.usp.br
}

Received 28 April 2009; Revised 22 June 2009; Accepted 15 July 2009

Recommended by Michael Wolin

We evaluated a relationship between nitric oxide plasma correlation and insulin resistance in white coat hypertensive patients. Patients were screened for white coat hypertension using an ambulatory blood pressure monitoring. The homeostasis model assessment insulin resistance index (HOMA-IR) was used to assess insulin resistance, and plasma nitrate concentrations were determined by chemiluminescence. The HOMA-IR was significantly higher in hypertensive $(3.84 \pm 0.62)$ when compared to white coat $(2.11 \pm 0.36)$ and normotensive $(1.40 \pm 0.21)$. We found no correlation between HOMA-IR and plasma nitrate levels in all three groups. We suggest that the more important issue for these patients is to focus on the metabolic abnormalities where lifestyle interventions such as weight control and exercise have proven effective.

Copyright () 2009 L. M. Marchi-Alves and E. C. Carnio. This is an open access article distributed under the Creative Commons Attribution License, which permits unrestricted use, distribution, and reproduction in any medium, provided the original work is properly cited.

\section{Introduction}

Insulin resistance plays a role in type 2 diabetes and is close related with public health problems, as obesity, dyslipidemias, coronary artery disease, hypertension, and a bunch of metabolic and cardiovascular malfunction which is defined as metabolic syndrome. Insulin resistance is an alteration in the action of insulin since besides the normal levels of insulin it cannot trigger the signal for glucose absorption. The effects of this condition can have profound pathophysiologic effects on various organs and tissues of the body. The clinical consequences, beyond hypertension, include hyperglycemia-induced tissue damage, dyslipidemia, metabolic syndrome, and cardiovascular disease [1].

Endothelium has many different roles including the regulation of blood-tissue permeability and vascular tonu, and control of vascular surface properties for during inflammation. Nitric oxide (NO) is a gaseous modulator, which acts in regulation of endothelial functions. An NO deficiency, which is also known as endothelial dysfunction, is the first step for the occurrence of many diseases including heart failure, diabetes mellitus, dyslipidemia, insulin resistance, and hypertension [2].
White coat hypertension is a well-known clinical situation defined as a persistently elevated blood pressure in the physician's office, even though the blood pressure is normal in other situations, and its prognostic significance remains controversial $[3,4]$. Whether individuals with WCH have an abnormal autonomic-cardiac regulation similar to that observed in sustained hypertensive patients is unknown, and in subjects with white coat hypertension it is unclear whether ambulatory blood pressure progresses over time and exhibits an increased cardiovascular risk. Additionally, it remains unclear whether white coat hypertension is already associated with vascular end-organ damage.

Some reports have suggested that white coat hypertension might be associated with someof these metabolic disturbances, instead of white coat hypertension per se, that may potentially explain the greater extent of end-organ damage sometimes observed in white coat hypertension patients when compared with normotensive individuals [5].

The combination of insulin resistance and compensatory hyperinsulinemia increases the likelihood that an individual will be hypertensive. Given the rapid increase in the number of clinical syndromes and abnormalities associated with 
insulin resistance/hyperinsulinemia, it seems reasonable to suggest that the cluster of these changes could be related to the defect in insulin action, which can be subsumed under the term of the insulin resistance syndrome. In addition to the identification of additional clinical syndromes related to insulin resistance/hyperinsulinemia, a number of new risk factors have been recognized that would increase cardiovascular risk in these individuals. Evidence is also accumulating that sympathetic nervous system activity is increased in insulin resistant, hyperinsulinemic individuals, and, along with the salt sensitivity associated with insulin resistance/hyperinsulinemia, increases the likelihood that these individuals will develop essential hypertension [6].

The ability of insulin to stimulate glucose disposal varies more than sixfold in apparently healthy individuals [7].

Overweight and obesity are highly associated with multiple comorbidities, such as elevated blood pressure values, dyslipidaemia, reduced insulin sensitivity, and alterations of large and minor vessels. Abdominal obesity is a risk factor for cardiovascular disease worldwide, and it is becoming a dramatic issue for national health systems [8].

The more overweight/obese the persons are, the more likely they are to be insulin-resistant and at increased risk of cardiovascular disease, but substantial numbers of overweight/obese individuals remain insulin-sensitive, and not all insulin-resistant persons are obese. Of greater clinical relevance is evidence that the metabolic benefit and decrease in risk of cardiovascular disease following weight loss occurs primarily in those overweight/obese individuals that are also insulin-resistant [6].

On the other hand, cardiovascular diseases are the most common causes of deaths, particularly in developed countries, and most are related with HT. Thus, BP control is the basis for the prevention of cardiovascular death. However, the diagnosis and management of hypertension is difficult due to the fact that $\mathrm{BP}$ varies greatly depending on physical and mental stresses [9].

Insulin resistance, obesity, and endothelial dysfunction are observed in some patients suffering from hypertension. It remains unclear whether patients with white coat hypertension elude the risk of this condition; so in this study we evaluated a relationship between nitrate plasma correlation and insulin resistance in white coat hypertension patients.

\section{Materials and Methods}

2.1. Subjects. Based on BP measurements at the medical office and on Ambulatory Blood Pressure Monitoring (ABPM), the patients were divided into the following categories: (a) sustained hypertension-systolic BP at the medical office $\geq 140 \mathrm{mmHg}$ or diastolic $\mathrm{BP} \geq 90 \mathrm{mmHg}$, or both, and mean of systolic and diastolic BP on ABPM during the wakefulness period $\geq 135 \mathrm{mmHg}$ and $\geq 85 \mathrm{mmHg}$, respectively; (b) white coat hypertension-systolic BP at the medical office $\geq 140 \mathrm{mmHg}$ or diastolic $\mathrm{BP} \geq 90 \mathrm{mmHg}$, or both, and mean of systolic and diastolic BP on ABPM during the wakefulness period $<135 \mathrm{mmHg}$ and $<85 \mathrm{mmHg}$, respectively; (c) normotension-systolic BP at the medical office $<140 \mathrm{mmHg}$ and diastolic $\mathrm{BP}<90 \mathrm{mmHg}$, and mean of systolic and diastolic BP on ABPM during the wakefulness period $<135 \mathrm{mmHg}$ and $<85 \mathrm{mmHg}$, respectively.

Exclusion criteria for entry in the study were proceeding from the pediatric clinic, pregnant patients, insulin using diabetics, and patients with devastating illnesses including malignancies, renal failure, liver diseases, and heart failure.

The procedures followed were in accordance with the ethical standards of the responsible committee on human experimentation and with the Helsinki Declaration and were approved by the Institutional Review Committee. Subjects gave informed consent. Study population came from the same geographic area.

2.2. Office BP Measurements. At the medical office, BP was measured with a regularly checked automate oscillometric device (OMRON) with an appropriate cuff for the arm's circumference. After a 5-minute rest with the patient seated, the measurements were taken on the patient's bare right arm, which was supported and maintained at the heart's level. The average of three measurements was taken as the mean systolic and diastolic pressures.

2.3. Ambulatory BP Monitoring (ABPM). ABPM was performed with an oscillometric device (SpaceLabs 90207), which was checked monthly against a mercury column sphygmomanometer. The measurements were taken every 15 minutes from 7:00 AM to 6:00 PM and every 30 minutes from 6:01 PM to 6:59 $\mathrm{AM}$, and the patient maintained his usual activities throughout the day. The appropriate cuff for the arm's circumference was placed on the no dominant arm, and the patients were instructed to maintain their arm stretched along their body and not to move their arm during measurements. The recording valid for analysis had a minimum duration of 24 hours and 80 valid readings, corresponding to at least $80 \%$ of all measurements.

2.4. Anthropometric Parameters. The anthropometric indicators were analyzed according to the criteria of World Health Organization-WHO [10]. Anthropometric measurements included weight, height, body mass index (BMI), and waist circumference. Every subject's height was measured in centimeters while the participant stood still without shoes, and weight was measured to the nearest $0.1 \mathrm{~kg}$ with electronic weight scale in kilograms with the participant lightly clothed. Subjects were categorized according to their body mass index (BMI). BMI was calculated as weight divided by square of height $\left(\mathrm{kg} / \mathrm{m}^{2}\right)$. Underweight was defined as a BMI less than $18.5 \mathrm{~kg} / \mathrm{m}^{2}$, normal weight as $18.5-24.9$, overweight as $25-$ $29.9 \mathrm{~kg} / \mathrm{m}^{2}$, and obesity as a BMI of $30.0 \mathrm{~kg} / \mathrm{m}^{2}$ or greater. Waist circumference (WC) was measured in centimeters at the midpoint between the bottom of the ribs and the top of the iliac crest. The cutoff points for cardiovascular disease risk was $102 \mathrm{~cm}$ for men and 88 for women.

2.5. Laboratory Methods. Subjects reported to the laboratory between 07:00 and 09:00 A.M., rested quietly for 10 minutes in the supine position, and a blood sample for the 
TABLE 1: Clinical characteristics of normotensive (NT), hypertensive (HT), and white coat hypertensive (WCH) patients.

\begin{tabular}{lccc}
\hline & NTs $(n=22)$ & HTs $(n=22)$ & WCHs $(n=20)$ \\
\hline Clinic Systolic BP (mmHg) & $114.9 \pm 2.8$ & $154.2 \pm 3.1^{\ddagger}$ & $142.1 \pm 1.75^{\ddagger \S}$ \\
Clinic Diastolic BP (mmHg) & $76.1 \pm 0.8$ & $98.6 \pm 2.1^{\ddagger}$ & $90.21 \pm 3.4^{\ddagger \S}$ \\
Ambulatory Systolic BP (mmHg) & $102.9 \pm 8.5$ & $143.9 \pm 3.9^{\ddagger}$ & $118.5 \pm 1.4^{\ddagger \#}$ \\
Ambulatory Diastolic BP (mmHg) & $71.79 \pm 1.24$ & $92.21 \pm 3.0^{\ddagger}$ & $75.78 \pm 1.44^{\ddagger \#}$ \\
Age (years) & $43.95 \pm 2.66$ & $49.14 \pm 3.42$ & $44.07 \pm 3.39$ \\
BMI $\left(\mathrm{kg} / \mathrm{m}^{2}\right)$ & $24.40 \pm 0.73$ & $29.13 \pm 1.02^{\ddagger}$ & $28.21 \pm 0.73^{\dagger}$ \\
WC $(\mathrm{cm})$ & $0.86 \pm 0.02$ & $0.92 \pm 0.02^{\dagger}$ & $0.89 \pm 0.02$ \\
\hline
\end{tabular}

${ }^{*} P<.05 \times \mathrm{NTs} ;{ }^{\dagger} P<.01 \times \mathrm{NTs},{ }^{\ddagger} P<.001 \times \mathrm{NTs},{ }^{\S} P<.05 \times \mathrm{HTs},{ }^{\sharp} P<.001 \times$ HTs.

determination of glucose and insulin was obtained from an antecubital vein and collected into a specific tube. Blood samples were drawn after a fasting period of 12 hours at the same time of the day. Serum collected was sent to a certified medical laboratory for subsequent analysis using automated enzymatic procedures with calculated precision values $<3 \%$. An estimation of insulin resistance was calculated using the homeostasis model analysis (HOMA-IR) using the formula: glucose $(\mathrm{mU} / \mathrm{L}) \times$ [insulin $(\mathrm{pmol} / \mathrm{L}) / 22.5]$ [11] .

Blood samples for nitrate determination were centrifuged immediately at $3000 \mathrm{~g}$ for 15 minutes, and supernatant was collected and frozen at $-20^{\circ} \mathrm{C}$ until assayed. Since $\mathrm{NO}$ is stoichiometrically converted to nitrate and nitrite, the levels of the NO produced in a response can be tracked by assaying for nitrate and nitrite. The NO levels were measured after enzymatic conversion of all nitrate to nitrite. Fasting peripheral venous blood samples for nitrate were collected into heparinized vacutainer tubes. Plasma samples were isolated by $2500 \mathrm{~g}$ centrifugation at $4^{\circ} \mathrm{C}$ for 10 minutes. All plasma samples were kept at $-70^{\circ} \mathrm{C}$ until the experiments were performed.

On the day of the assay, plasma samples were thawed and deproteinized with $95 \%$ ethanol $\left(\right.$ at $\left.4^{\circ} \mathrm{C}\right)$ for 30 minutes, subsequently centrifuged, and the supernatant was used for measurement of nitrate according to the NO/ozone chemiluminescence technique as previously described by Archer [12], using a Sievers NO Analizer (Sievers 280 NOA; Sievers, Boulder, CO). Sodium nitrate (Sigma Chemical) was used as a standard reference.

2.6. Statistical Method. The values of the selected variables were defined as mean \pm SD. The NO values of the groups (normotensive, hypertensive, and white coat hypertensive) were compared with one-way ANOVA (Tukey HSD). "P" values $<.05$ were considered statistically significant. Pearson correlation analysis was used to find out the correlations between HOMA-IR and nitrate plasma concentration.

\section{Results}

In this study 71 patients were screened for white coat hypertension, of which 64 were included in the study. The study group consisted of 20 subjects with white coat hypertension (30\% male and 70\% female patients), 22

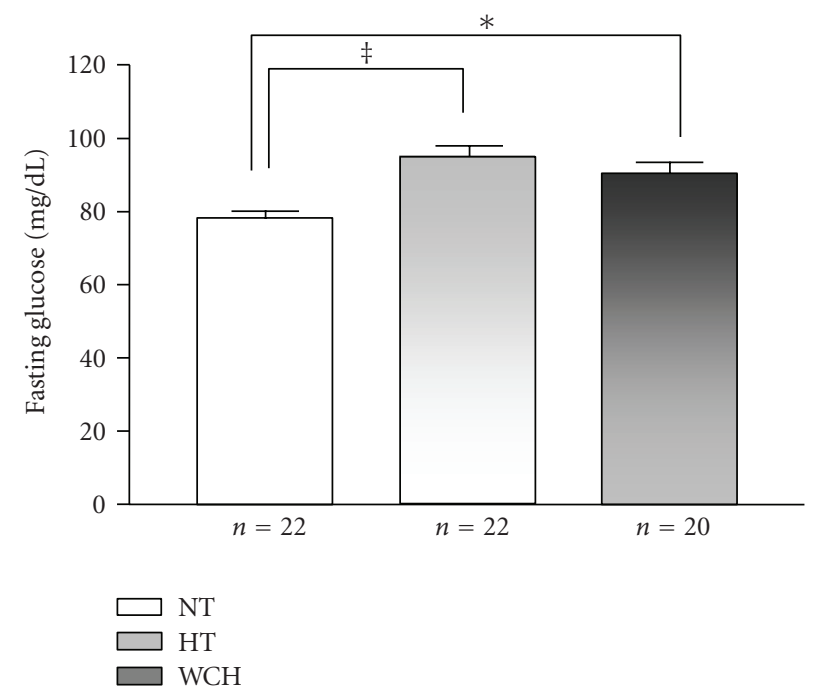

Figure 1: Fasting glucose levels $(\mathrm{mg} / \mathrm{dL})$ in normotensive (NT), sustained hypertensive $(\mathrm{HT})$, and white coat hypertensive $(\mathrm{WCH})$ patients. ${ }^{\ddagger} P<.001,{ }^{*} P<.05$.

hypertensives (68.2\% male and $31.8 \%$ female patients), and 22 normotensive participants (31.8\% male and $68.2 \%$ female patients).

Clinical characteristics of subjects of the three groups are listed in Table 1. From the total $27.27 \%$ of normotensives, $31.82 \%$ of hypertensives and $28.57 \%$ of white coat hypertensives were overweight. The occurrence of obesity was $9.09 \%$ of normotensives, $50.62 \%$ of hypertensives, and $14.28 \%$ of white coat hypertensives.

Plasma glucose levels of the three groups are shown in Figure 1. The HTs $(95.0 \pm 3.26 \mathrm{mg} / \mathrm{dL})$ and white coat hypertensives $(90.07 \pm 3.72 \mathrm{mg} / \mathrm{dL})$ had significantly higher levels of glucose than the normotensives (78.14 \pm $2.05 \mathrm{mg} / \mathrm{dL}$ ). No significant difference was observed between white coat hypertensives and hypertensives regarding these parameters.

Figure 2 shows fasting plasma insulin concentrations in all three groups tested. There was a statistically significant difference $(P<.01)$ between the hypertensive group $(17.10 \pm 0.91 \mu \mathrm{U} / \mathrm{mL})$ and the normotensive group (6.99 \pm $0.91 \mu \mathrm{U} / \mathrm{mL}$ ). In addition, there was no significant difference regarding fasting plasma insulin concentrations between 


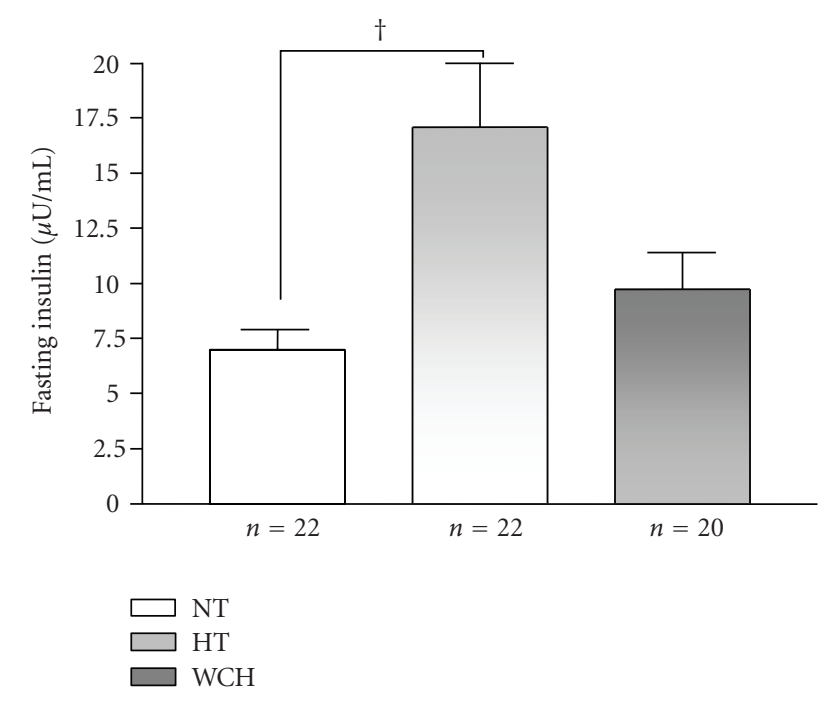

FIgURE 2: Fasting insulin levels $(\mu \mathrm{U} / \mathrm{ml})$ in normotensive (NT), sustained hypertensive (HT), and white coat hypertensive $(\mathrm{WCH})$ patients. ${ }^{\dagger} P<.01$.

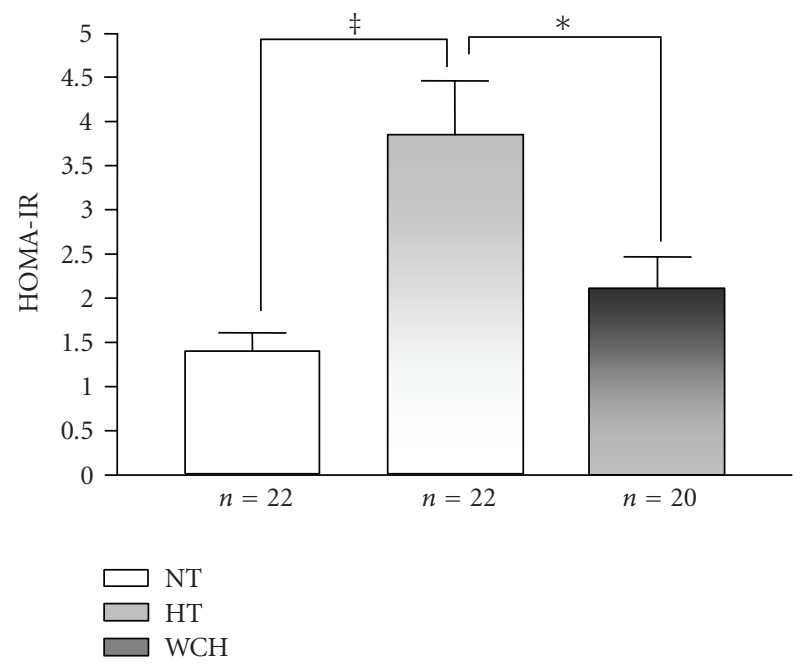

FIGURE 3: HOMA-IR in normotensive (NT), sustained hypertensive (HT), and white coat hypertensive (WCH) patients. ${ }^{\ddagger} P<.001,{ }^{*} P<$ .05 .

the white coat hypertensives $(9.73 \pm 1.68 \mu \mathrm{U} / \mathrm{mL})$ and the normotensives or hypertensives groups.

The HOMA-IR values in the groups are demonstrated in Figure 3. The values was significantly higher in hypertensives $(3.84 \pm 0.62)$ when compared to white coat hypertensives $(2.11 \pm 0.36)$ and normotensives $(1.40 \pm 0.21)$. There were no significant differences between white coat hypertensives and normotensives.

Figure 4 shows the correlation between HOMA-IR and nitrate plasma concentration. We found no correlation between HOMA-IR and plasma nitrate levels in all three different groups.

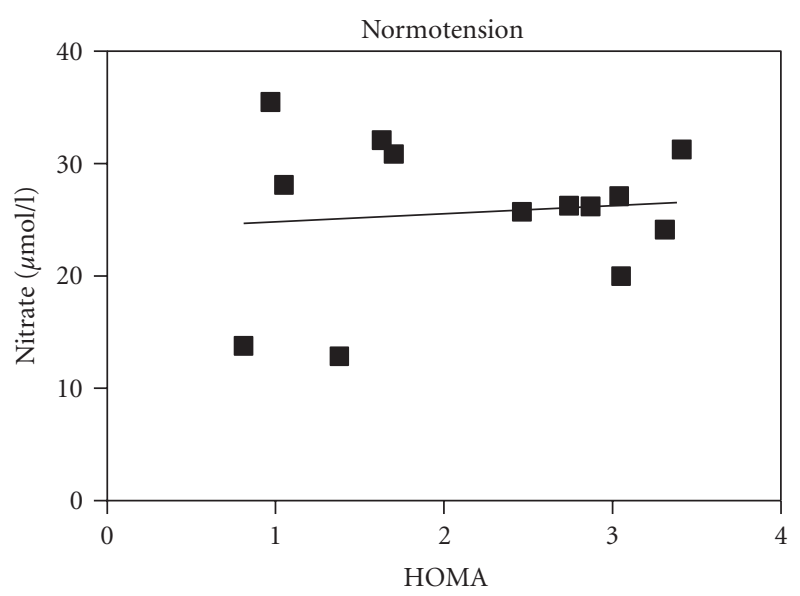

(a)

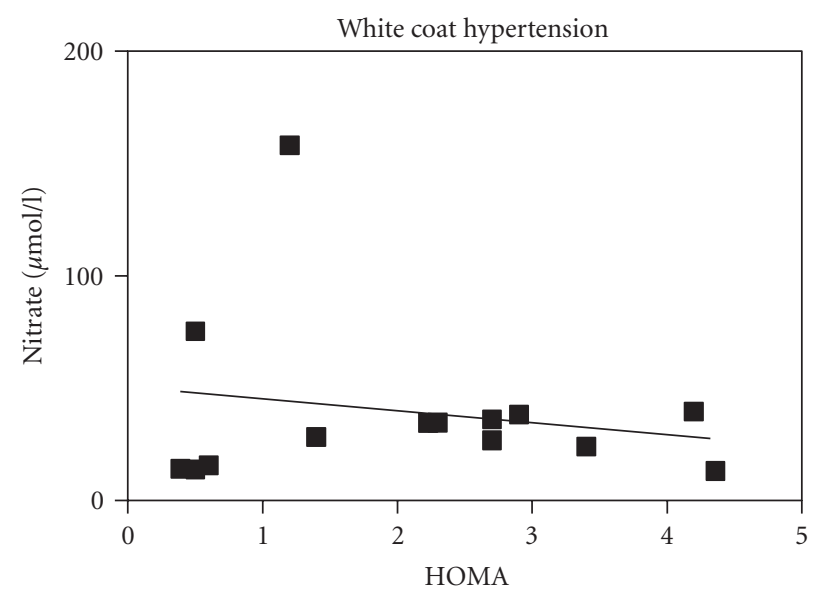

(b)

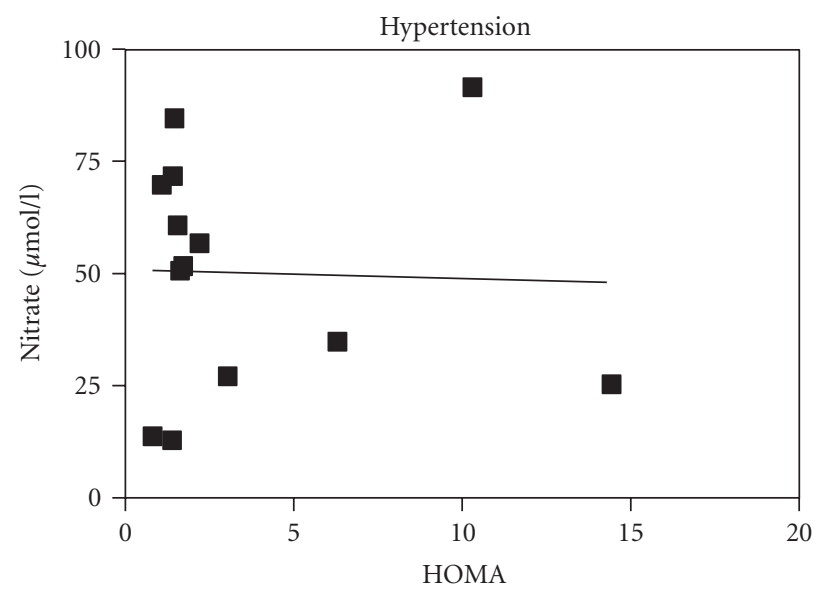

(c)

FIgURE 4: Relationship between HOMA and plasma nitrate levels in normotensive (NT), sustained hypertensive (HT), and white coat hypertensive $(\mathrm{WCH})$ patients.

\section{Discussion}

The results of this study demonstrate a normal insulin resistance in white coat hypertensive patients.

Although the white coat hypertensive group had similar fasting insulin levels compared with normotensive group, 
careful inspection of their data shows that their white coat hypertension population had characteristics of the metabolic syndrome (increased body mass index and fasting glucose level), which were also present in patients with sustained hypertension.

Although the majority of individuals in the general population that can be considered insulin resistant are also overweight/obese, not all overweight/obese persons are insulin resistant. Of greater clinical relevance is the fact that significant improvement in these metabolic abnormalities following weight loss is seen only in the subset of overweight/obese individuals that are also insulin resistant. In view of the large number of overweight/obese subjects at potential risk to be insulin resistant/hyperinsulinemic (and at increased cardiovascular risk), and the difficulty in achieving weight loss, it seems essential to identify those overweight/obese individuals who are also insulin resistant and will benefit the most from weight loss, then target this population for the most-intensive efforts to bring about weight loss [7].

In relation to results of HOMA IR, researchers assumed different methods to describe a threshold, such as the top quintile and 90th percentile of the HOMA in normal glucose tolerant nonobese populations. Considering the patients whose HOMA-IR is above the 90th percentile as insulin resistant subjects, Geloneze et al. [13] found a threshold value for insulin resistance of 2.71. In accordance with these criteria, inside of this proposal, our findings determine that the insulinoresistance is present in the carriers of essential hipertensive and normotensive individuals a presence of indices of normality in the evaluation of this parameter. But, in interesting way, when we evaluate the group white coat hypertension, even so let us not find statistically difference between normotension, we observe raised indices, that do not allow to fit them in the category of normoglycemics [13]. However, to validate the potential use of HOMA-IR values in clinical practice, it is important to establish its distribution in a sample of normoglycemic individuals from a population with the same genetic background [14]. In this regard, Ghiringhello et al. [15] describe the distribution of HOMA-IR values in a large sample of Brazilian population $(1.2 \pm 0.65$ para $\mathrm{IMC}<25,1.8 \pm 0.98$ para $\mathrm{IMC}=25-30$ e $2.9 \pm$ 1.6 para IMC > 30). Therefore, if we calculated the HOMAIR threshold in those subjects who fulfilled this criteria, we would find that the essential hypertensive subjects as well as the white coat hypertensives present a higher index than that observed at the normal criteria, having had to be categorized as insulin-resistant. That is, if we considered this criterion of definition for insulin-resistance determination, the results point that the insulin resistance is definitively installed in white coat hypertension [15].

Although stated that the white coat hypertension group had similar fasting insulin and HOMA-IR results, compared to normotensives, careful inspection of our data shows that their white-coat hypertension population had characteristics of the insulin-resistance syndrome: elevated body mass index, fasting glucose levels, and estimated insulin resistance. Thus, we agree to previous studies that they indicate a possible relationship between end-organ damage and insulin resistance in patients with white coat hypertension exists [16, 17]. The association between white-coat hypertension and metabolic disturbances of insulin-resistance syndrome may help explain the high prevalence of cardiovascular disease often associated with white coat hypertension [18-20].

White coat hypertension and metabolic abnormalities characteristic of insulin-resistance syndrome coexist, and in which the increased risk of target organ damage is determined not by the BP, but by the metabolic abnormalities. This distinction would account for some previous findings of increased target organ damage in white coat hypertension [20].

\section{Conclusions}

White coat hypertension should preferentially be accepted as an alarming sign of excess weight and many associated disorders in the future, rather than just being considered a predisposing factor of hypertensive or atherosclerosis alone [21]. There are limitations in this study, which was conducted on a small sample of patients, and the subjects are relatively young with no inclusion criteria. Thus, although no hypertension-related target organ damage was detected in the present study, white coat hypertension cannot be regarded as a benign phenomenon because these subjects display metabolic abnormalities that are powerful risk factors for future atherosclerotic events. So in conclusion we suggest that the more important issue for the patients is to focus on the metabolic abnormalities related to the insulinresistance syndrome where lifestyle interventions such as weight control and exercise have proven effective. However we fail to prove that it could be a possible interaction between a decreased NO bioavailability, endothelial dysfunction, and the development of insulin resistance in white coat hypertensive patients.

Arterial hypertension remains a devastating disease. All current medical therapy is expensive, complex and largely palliative. The management of hypertensive patients requires co-ordination between the patient, their support system, and healthcare professionals. Contemporary practice requires responsibility and a role in the primary prevention, detection and treatment of hypertension [22]. Healthcare professionals need to combine scientific knowledge and practice to determine the prognosis and treatment of white coat hypertension, in an effort to provide the highest quality care to the patient [23].

\section{References}

[1] P. S. Jellinger, "Metabolic consequences of hyperglycemia and insulin resistance," Clinical Cornerstone, vol. 8, supplement 7, pp. S30-S42, 2007.

[2] K. Bian, M. F. Doursout, and F. Murad, "Vascular system: role of nitric oxide in cardiovascular diseases," Journal of Clinical Hypertension, vol. 10, no. 4, pp. 304-310, 2008.

[3] Y. Karter, A. Curgunlu, S. Altinişik, et al., "Target organ damage and changes in arterial compliance in white coat hypertension. Is white coat innocent?" Blood Pressure, vol. 12, no. 5-6, pp. 307-313, 2003. 
[4] J. J. Polonia, G. M. Gama, J. A. Silva, C. Amaral, L. R. Martins, and S. E. Bertoquini, "Sequential follow-up clinic and ambulatory blood pressure evaluation in a low risk population of white-coat hypertensive patients and in normotensives," Blood Pressure Monitoring, vol. 10, no. 2, pp. 57-64, 2005.

[5] G. Mulè, E. Nardi, S. Cottone, et al., "Metabolic syndrome in subjects with white-coat hypertension: impact on left ventricular structure and function," Journal of Human Hypertension, vol. 21, no. 11, pp. 854-860, 2007.

[6] G. Reaven, "All obese individuals are not created equal: insulin resistance is the major determinant of cardiovascular disease in overweight/obese individuals," Diabetes and Vascular Disease Research, vol. 2, no. 3, pp. 105-112, 2005.

[7] G. Reaven, F. Abbasi, and T. McLaughlin, "Obesity, insulin resistance, and cardiovascular disease," Recent Progress in Hormone Research, vol. 59, pp. 207-223, 2004.

[8] J. Segura and L. M. Ruilope, "Obesity, essential hypertension and renin-angiotensin system," Public Health Nutrition, vol. 10, no. 10, pp. 1151-1155, 2007.

[9] M. Ezzati, A. D. Lopez, A. Rodgers, S. Vander Hoorn, and C. J. L. Murray, "Selected major risk factors and global and regional burden of disease," The Lancet, vol. 360, no. 9343, pp. 13471360, 2002.

[10] World Health Organization, "Physical status: the use and interpretation of anthropometry. Report of a WHO Expert Comitee," Tech. Rep. series no 854, World Health Organization, Geneva, Switzerland, 1995.

[11] D. R. Matthews, J. P. Hosker, A. S. Rudenski, B. A. Naylor, D. F. Treacher, and R. C. Turner, "Homeostasis model assessment: insulin resistance and $\beta$-cell function from fasting plasma glucose and insulin concentrations in man," Diabetologia, vol. 28, no. 7, pp. 412-419, 1985.

[12] S. Archer, "Measurement of nitric oxide in biological models," FASEB Journal, vol. 7, no. 2, pp. 349-360, 1993.

[13] B. Geloneze, E. M. Repetto, S. R. Geloneze, M. A. Tambascia, and M. N. Ermetice, "The threshold value for insulin resistance (HOMA-IR) in an admixtured population. IR in the Brazilian Metabolic Syndrome Study," Diabetes Research and Clinical Practice, vol. 72, no. 2, pp. 219-220, 2006.

[14] T. M. Wallace, J. C. Levy, and D. R. Matthews, "Use and abuse of HOMA modeling," Diabetes Care, vol. 27, no. 6, pp. 14871495, 2004.

[15] M. T. Ghiringhello, J. G. H. Vieira, T. T. Tachibana, et al., "Distribution of HOMA-IR in Brazilian subjects with different body mass indexes," Arquivos Brasileiros de Endocrinologia e Metabologia, vol. 50, no. 3, pp. 573-574, 2006.

[16] A. Tunçkale, S. N. Aran, H. Karpuz, and A. Dirican, "Relationship between insulin resistance and end-organ damage in white coat hypertension," American Journal of Hypertension, vol. 17, no. 11, pp. 1011-1016, 2004.

[17] M. F. Muldoon, P. Nazzaro, K. Sutton-Tyrrell, and S. B. Manuck, "White-coat hypertension and carotid artery atherosclerosis: a matching study," Archives of Internal Medicine, vol. 160, no. 10, pp. 1507-1512, 2000.

[18] S. Julius, A. Mejia, K. Jones, et al., "“White coat" versus "sustained" borderline hypertension in Tecumseh, Michigan," Hypertension, vol. 16, no. 6, pp. 617-623, 1990.

[19] T. E. Strandberg and V. Salomaa, "White coat effect, blood pressure and mortality in men: prospective cohort study," European Heart Journal, vol. 21, no. 20, pp. 1714-1718, 2000.

[20] N. Zakopoulos, C. Papamichael, H. Papaconstantinou, et al., "Isolated clinic hypertension is not an innocent phenomenon: effect on the carotid artery structure," American Journal of Hypertension, vol. 12, no. 3, pp. 245-250, 1999.
[21] M. R. Helvaci, H. Kaya, A. Yalcin, and G. Kuvandik, "Prevalence of white coat hypertension in underweight and overweight subjects," International Heart Journal, vol. 48, no. 5, pp. 605-613, 2007.

[22] D. McLean, K. Kingsbury, J. A. Costello, L. Cloutier, and S. Matheson, "Hypertension Education Program (CHEP) recommendations: management of hypertension by nurses," Canadian Journal of Cardiovascular Nursing, vol. 17, no. 2, pp. 10-16, 2007.

[23] L. M. Alves, M. S. Nogueira, E. V. Veiga, S. de Godoy, and E. C. Carnio, "White coat hypertension and nursing care," Canadian Journal of Cardiovascular Nursing, vol. 13, no. 3, pp. 29-34, 2003. 


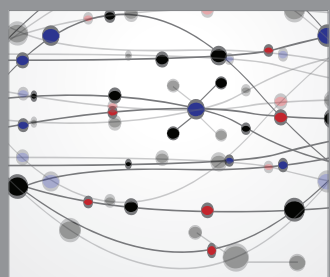

The Scientific World Journal
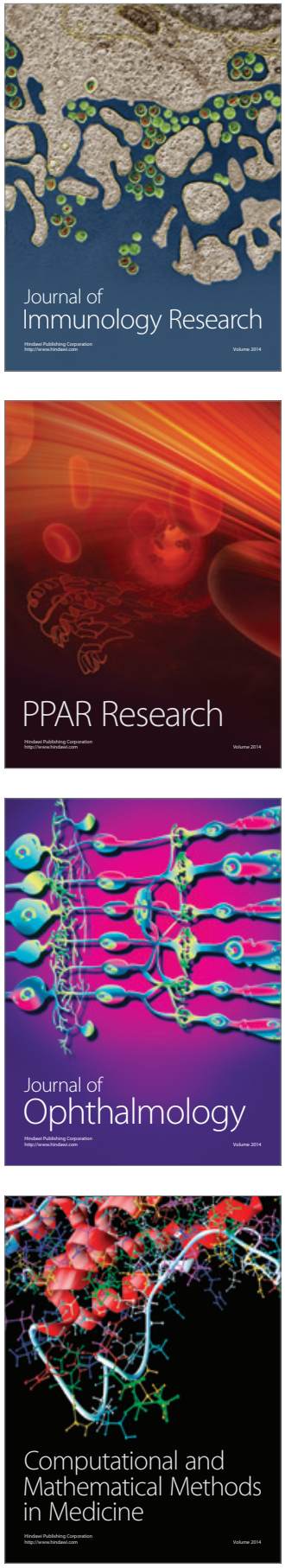

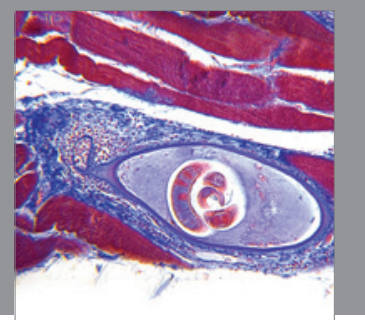

Gastroenterology

Research and Practice
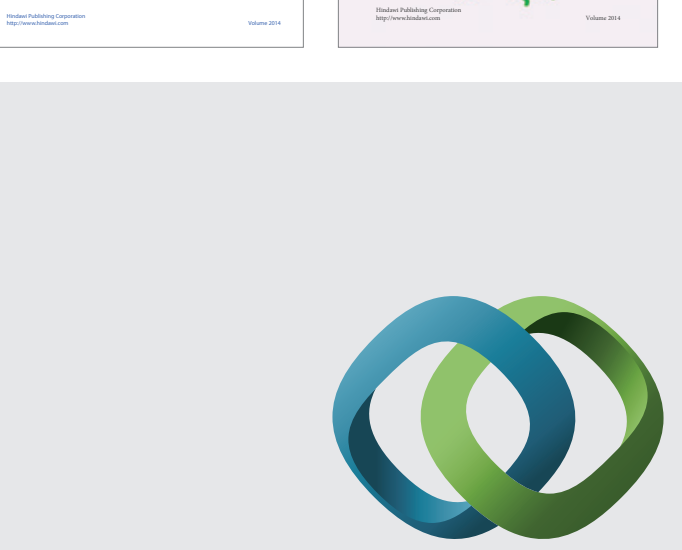

\section{Hindawi}

Submit your manuscripts at

http://www.hindawi.com
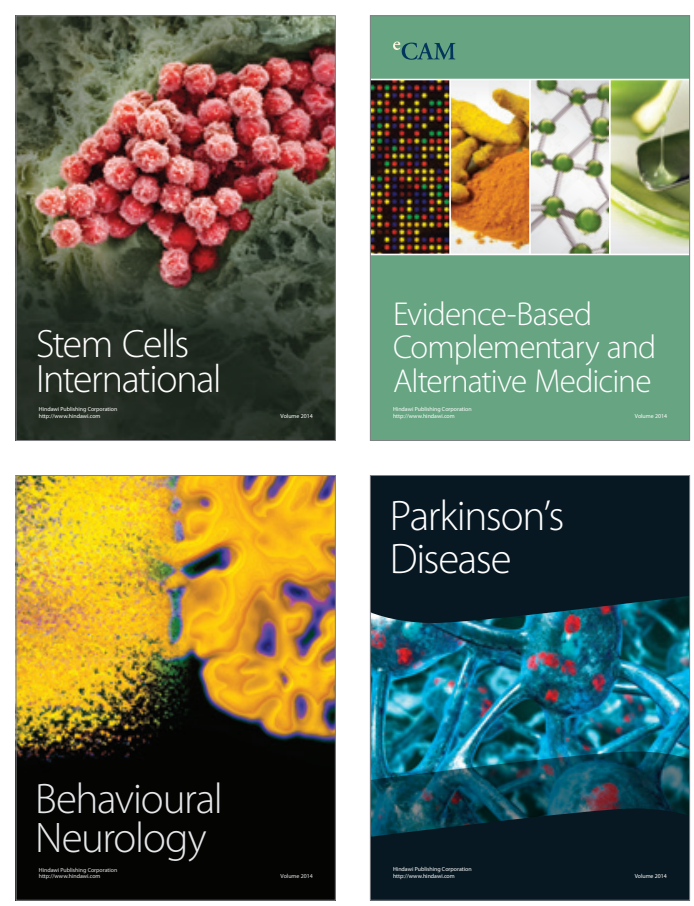

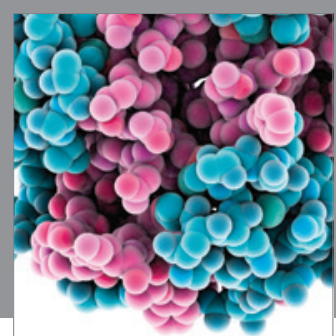

Journal of
Diabetes Research

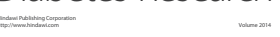

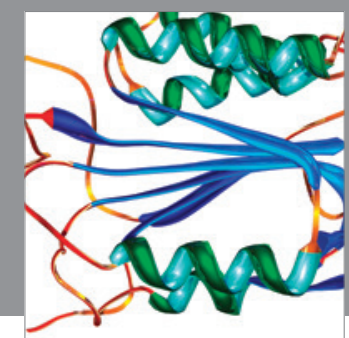

Disease Markers
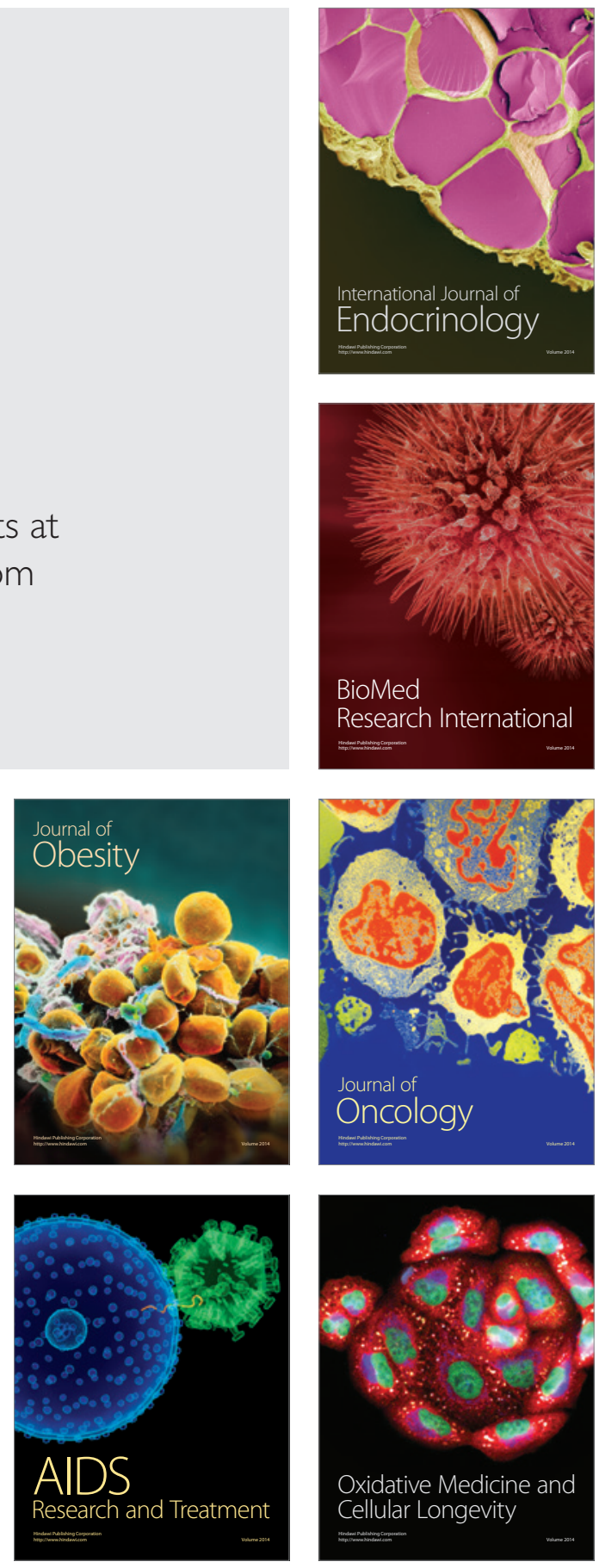